\title{
The Gate Distribution
}

\author{
Yoram Zimmels and Leonid G. Fel ${ }^{*}$ \\ Department of Civil and Environmental Engineering, Technion - Israel Institute of Technology, Haifa 32000, Israel
}

\begin{abstract}
Gates that transfer objects within their capacity, without formation of queues, are considered in a combinatorial framework. The number of different ways, $I(m, n, \ell), n$ objects can pass randomly an array of $m$ gates, each with constrained capacity $\ell$, is used to characterize its performance. Two gate distributions were formulated and their properties studied. The first, $n$ dependent distribution was found to be symmetric, whereas the second, $\ell$ dependent distribution is skew. These properties persist also in the case of multivariate gate distributions. In the latter case, the expectation and variance turn to have additive properties, respectively.
\end{abstract}

\section{INTRODUCTION AND STATEMENT OF PROBLEM}

Many service systems provide access or exist in the form of gates. This is typical of arrays of cashiers in large retail and discount stores, such as supermarkets and warehouses, cashiers and gates in stadiums and amusement parks, toll plazas, tellers in banks, security checks in airports, and services provided by local and national authorities to the public in municipality and government agencies. Gates are commonplace in computational hardware. Systems that transfer information and facilitate communication, e.g. telephone exchange centers, can be also viewed as transfer gates for incoming calls.

Devices for purification of materials often rely on differential capacity for mass transfer, whereby the slower material is separated across a barrier from the faster one. Gate systems can be characterized by their number, capacity per gate and variability, e.g. if there are different gates in the array. We define an ideal gate system as one that operates within its capacity range so that no queues, delays or blockage of passage can occur. This is an ideal situation that guarantees customer or user satisfaction, and hence must be the target of any advanced design. If the approach to the array of gates is random, then its performance is enhanced with an increase in the number of ways it can be passed within the limits of its capacity. Thus, we are interested in the conditions that provide the maximum number of ways in which an assembly of identical objects can pass the gate array randomly, without exceeding its capacity and avoiding the formation of queues.

We explore the sensitivity of the array to a unit change in the object transfer capacity per gate. We also consider heterogeneous arrays comprising sections of gates with different capacities. The studied gate systems is viewed in the context of two new distributions, which are functions of the number of gates, their specific capacity, and the number of passing objects or entities.

In the first gate distribution, the number of passing objects serves as the random variable, while the number of

*Address correspondence to this author at the Department of Civil and Environmental Engineering, Technion - Israel Institute of Technology, Haifa 32000, Israel; E-mail: lfel@tx.technion.ac.il gates and the capacity per gate are set as system parameters. In the second gate distribution, the capacity per gate serves as the random variable, while the number of gates and passing objects turn system parameters. The first gate distribution is shown to be symmetric for uniform as well as heterogeneous gate arrays. In contrast, the second gate distribution is skew, irrespective of the gate array being uniform or not. Furthermore, it is also self shifting with respect to the range wherein it is defined. The symmetry of the first gate distribution facilitates a good fit, in a wide range of the number of gates, capacity limit and passing objects, with the normal distribution having the same expectation and variance.

Finally, these gate distribution facilitate analysis and optimization of performance regarding passage of objects in a mode of queueless operations.

\section{OBJECTIVE OF WORK}

1. In how many ways, $I(m, n, \ell)$, can $n$ objects pass $m$ gates per unit time subject to the constraint that the maximum allowable capacity of each gate is $\ell$ objects per unit time. Note that the variable time can be replaced by any other measuring unit such as length and area, depending on the nature of the problem.

2. Identify and formulate the relevant distributions associated with $I(m, n, \ell)$, as a function of $m, n, \ell$, with respect to their significance and properties.

In item 1 , it is implied that as long as the capacity of a gate is not exceeded, there is no queuing. A queue is formed once the capacity is exceeded, but according to the definition of $I(m, n, \ell)$, this is not allowed.

\section{THEORY}

The expression of $I(m, n, \ell)$,

$I(m, n, \ell)=\sum_{i=0}^{\left\lfloor\frac{n}{\lambda}\right\rfloor}(-1)^{i}\left(\begin{array}{l}m \\ i\end{array}\right)\left(\begin{array}{c}m+n-i \lambda-1 \\ m-1\end{array}\right), \lambda=\ell+1, n \leq m \ell$

and the symmetry property $I(m, n, \ell)=I(m, m \ell-n, \ell)$ are derived in the Appendix. 
The number of ways, $Q(m, n, \ell)$, that a queue of up to $\mathrm{n}$ $\ell$ objects is formed in at least one gate, is expressed in Appendix by, $\quad \sum_{i=1}^{\lfloor n / \lambda\rfloor}(-1)^{i-1}\left(\begin{array}{c}m \\ i\end{array}\right)\left(\begin{array}{c}m+n-i \lambda-1 \\ m-1\end{array}\right)$. Clearly $I(m, n, \ell)+Q(m, n, \ell)=\left(\begin{array}{c}m+n-1 \\ m-1\end{array}\right)$.

Eq. (1) is the solution of an equivalent problem where $n$ objects are distributed in $m$ boxes that can hold up to $\ell$ objects per box $[1,2]$. In this case, $I(m, n, \ell)$ is the number of different ways the $n$ objects can be distributed in the $m$ boxes. Note that if $n>m \ell$ or $n<0$, then $I(m, n, \ell)=0$.

The first gate distribution is defined as

$g_{n}(m, n, \ell)=I(m, n, \ell) / \sum_{i=0}^{m \ell} I(m, i, \ell), 0 \leq n \leq m \ell$

where $g_{n}(m, n, \ell)$ vanishes outside the range $[0, m \ell]$, and $m \ell$ is the maximum total capacity of all gates to pass objects per unit time. This distribution is a function of $n$, at fixed $m$ and $\ell$. The second gate distribution is,

$G_{\ell}(m, n, \ell)=I(m, n, \ell) / I(m, n, n), \frac{n}{m}-1 \leq \ell \leq n$

Unlike $g_{n}(m, n, \ell), G_{\ell}(m, n, \ell) \quad$ is a cumulative distribution ranging from 0 to 1 . Taking its discrete derivative gives

$g_{\ell}(m, n, \ell)=\frac{1}{I(m, n, n)}[I(m, n, \ell+1)-I(m, n, \ell)]$,

$\frac{n}{m}-1 \leq \ell \leq n$

Observe that $I(m, n, n)=\left(\begin{array}{c}m+n-1 \\ m-1\end{array}\right)$, which is the number of different ways the objects can pass the $m$ gates per unit time at $\ell=n$, i.e., in the case there is no constraint on the gate capacity.

The functions $g_{n}(m, n, \ell)$ and $G_{\ell}(m, n, \ell)$ can be included in the list of discrete distributions known in literature [3].

In the case of multivariate ( $k$ groups) problem, $\bar{m}=\left(m_{1}, \ldots, m_{k}\right)$ and $\bar{\ell}=\left(\ell_{1}, \ldots, \ell_{k}\right), \quad g_{n}(\bar{m}, n, \bar{\ell}) \quad$ stays symmetric,

$$
I(\bar{m}, n, \bar{\ell})=I(\bar{m}, L-n, \bar{\ell}), L=\sum_{i=1}^{k} L_{i}, L_{i}=m_{i} \ell_{i},
$$

where, $L_{i}$ and $\mathrm{L}$ are partial and total capacity of $m_{i}$ and $m=\sum_{i=1}^{k} m_{i}$ gates, respectively. In this case, $g_{n}(\bar{m}, n, \bar{\ell})$ can be evaluated by a recurrence convolution formula.
Define $\Psi(\bar{m}, \bar{\ell}, t)$ as a product of $k$ generating functions $[1,2,4], \psi\left(m_{i}, \ell_{i}, t\right)$,

$$
\begin{aligned}
& \Psi(\bar{m}, \ell, t)=\prod_{i=1}^{k} \psi\left(m_{i}, \ell_{i}, t\right), \psi\left(m_{i}, \ell_{i}, t\right) . \\
& =\left(1+t+\ldots+t^{\ell_{i}}\right)^{m_{i}}
\end{aligned}
$$

Following a transformation we have,

$$
\begin{aligned}
& \Psi(\bar{m}, \bar{\ell}, t)=t^{L} \prod_{i=1}^{k}\left(1+t^{-1}+\ldots+t^{-\ell_{i}}\right)^{m_{i}} \\
& =z^{-L} \prod_{i=1}^{k}\left(1+z+\ldots+z^{\ell_{i}}\right)^{m_{i}}, z=t^{-1}
\end{aligned}
$$

Express $\Psi$ as a polynomial in $z$,

$$
\begin{aligned}
& \Psi(\bar{m}, \bar{\ell}, t)=z^{-L} \sum_{n=0}^{L} I(\bar{m}, n, \bar{\ell}) z^{n} \\
& \sum_{n=0}^{L} I(\bar{m}, n, \bar{\ell}) t^{L-n}=\sum_{n=0}^{L} I(\bar{m}, L-n, \bar{\ell}) t^{n}
\end{aligned}
$$

The corresponding polynomial of eq. (6) is

$$
\Psi(\bar{m}, \bar{\ell}, t)=\sum_{n=0}^{L} I(\bar{m}, n, \bar{\ell}) t^{n}
$$

Comparing eqs. (8) and (9) gives eq. (5). Note that if the number of objects $n_{i}$ passing the $m_{i}$ gates exceeds $m_{i} \ell_{i}$, its contribution to $I(\bar{m}, n, \bar{\ell})$ vanishes, $n=\sum_{i=1}^{k} n_{i}$.

The distribution of $n$ objects among the $k$ groups of gates, where the maximum capacity of the ith group is $L_{i}$ can be done in $I(\overline{1}, n, \bar{L})$ ways, $\overline{1}=(1, \ldots, 1), \bar{L}=\left(L_{1}, \ldots, L_{k}\right)$.

Eq. (A10) in appendix follows from eq. (5) at $k=1$, and by virtue of eq. (2), $g_{n}(m, n, \ell)$ is also symmetric as its value remains unchanged when $n$ and $i$ are replaced by $m \ell-n$ and $m \ell-I$, respectively. Using eq. (5), the same applies to a multivariate distribution $g_{n}(\bar{m}, n, \bar{\ell})$.

Expectation $E_{k}(n)$ and Variance $V_{k}(n)$ of the multivariate gate distribution.

Expanding $\psi\left(m_{i}, \ell_{i}, t\right)$ and $\Psi(\bar{m}, \bar{\ell}, t)$ as polynomials in $t$ gives 


$$
\begin{aligned}
& \Psi\left(m_{i}, \ell_{i}, t\right)=\sum_{n=0}^{L_{i}} I\left(m_{i}, n, \ell_{i}\right) t^{n}, \\
& \Psi(\bar{m}, \bar{\ell}, t)=\sum_{n=0}^{L} I(\bar{m}, n, \bar{\ell}) t^{n},
\end{aligned}
$$

where, $I\left(m_{i}, n, \ell_{i}\right)$ is defined in eq. (1) and $I(\bar{m}, n, \bar{\ell})$ stands for its multivariate counterpart.

Substitute eqs. (10) and (11) in eq. (6) and obtain

$$
I(\bar{m}, n, \bar{\ell})=\sum_{n_{1}+\ldots+n_{k}=n} \prod_{i=1}^{k} I\left(m_{i}, n_{i}, \ell_{i}\right) .
$$

Equality (12) expresses $I(\bar{m}, n, \bar{\ell})$ as a convolution of $I\left(m_{i}, n, \ell_{i}\right) . \quad$ In what follows, we calculate $E_{k}(n)$ and $V_{k}(n)$ for the multivariate case.

$$
\begin{aligned}
& E_{k}(n)=\frac{1}{\Psi(t=1)} \sum_{n=0}^{L} n I(\bar{m}, n, \bar{\ell}) \\
& V_{k}(n)=\frac{1}{\Psi(t=1)} \sum_{n=0}^{L} n^{2} I(\bar{m}, n, \bar{\ell})-\left[E_{k}(n)\right]^{2}
\end{aligned}
$$

where, $\Psi=\Psi(\bar{m}, \bar{\ell}, t)$ is used for the sake of brevity. Differentiating eqs. (6) and (11), with respect to $t$, gives

$$
\Psi^{\prime} / \Psi=\sum_{i=1}^{k} \psi_{i}^{\prime} / \Psi_{i}, \Psi^{\prime}=\sum_{n=0}^{L} n I(\bar{m}, n, \bar{\ell}) t^{n-1}
$$

where, $\psi_{i}=\psi\left(m_{i}, \ell_{i}, t\right)$. Combining eqs. (13) and (14) gives

$$
E_{k}(n)=\Psi^{\prime}(t=1) / \Psi(t=1)=\sum_{i=0}^{k} \psi_{i}^{\prime}(t=1) / \psi_{i}(t=1)(16)
$$

Calculating $\psi_{i}^{\prime}$ gives

$$
\psi_{i}^{\prime}=m_{i}\left(1+t+\ldots+t^{\ell}\right)^{m_{i}-1} \sum_{r=0}^{\ell_{i}} r t^{r-1}
$$

so that

$$
\psi_{i}^{\prime}(t=1) / \psi_{i}(t=1)=\frac{m_{i}}{\ell_{i}+1} \sum_{r=0}^{\ell_{i}} r=\frac{m_{i} \ell_{i}}{2},
$$

and finally we get

$$
E_{k}(n)=\frac{1}{2} \sum_{i=1}^{k} m_{i} \ell_{i}
$$

This is in agreement with the symmetry properties of $I(\bar{m}, n, \bar{\ell})$ as per eq. (5). Differentiation of $\Psi^{\prime}$ in eq. (15) gives

$$
\Psi^{\prime \prime}=\sum_{n=0}^{L} n^{2} I(\bar{m}, n, \bar{\ell}) t^{n-2}-\sum_{n=0}^{L} n I(\bar{m}, n, \bar{\ell}) t^{n-2}
$$

Recalling eq. (15), we get

$$
\frac{1}{\Psi(t=1)} \sum_{n=0}^{L} n^{2} I(\bar{m}, n, \bar{\ell})=\frac{\Psi^{\prime \prime}(t=1)}{\Psi(t=1)}+\frac{\Psi^{\prime}(t=1)}{\Psi(t=1)} .
$$

Thus, by eqs. (14), (16) and (20),

$$
V_{k}(n)=\frac{\Psi^{\prime \prime}(t=1)}{\Psi(t=1)}+\frac{\Psi^{\prime}(t=1)}{\Psi(t=1)}-\left[\frac{\Psi^{\prime}(t=1)}{\Psi(t=1)}\right]^{2}
$$

Differentiation of the left-hand-side equality in eq. (15) gives,

$$
\frac{\Psi^{\prime \prime}(t=1)}{\Psi(t=1)}=\left[\frac{\Psi^{\prime}(t=1)}{\Psi(t=1)}\right]^{2}+\sum_{i=1}^{k}\left[\frac{\psi_{i}^{\prime \prime}}{\psi_{i}}-\left(\frac{\psi_{i}^{\prime}}{\psi_{i}}\right)^{2}\right]_{t=1}
$$

Combining eqs. (21) and (22) gives,

$$
V_{k}(n)=\sum_{i=0}^{k}\left[\frac{\psi_{i}^{\prime}}{\psi_{i}}+\frac{\psi_{i}^{\prime \prime}}{\psi_{i}}-\left(\frac{\psi_{i}^{\prime}}{\psi_{i}}\right)^{2}\right]_{t=1}
$$

Calculation of the summand in eq. (23) finally gives,

$$
V_{k}(n)=\sum_{i=1}^{k} \frac{m_{i} \ell_{i}\left(\ell_{i}+2\right)}{12}
$$

Eqs. (21) and (24) show that both $E_{k}(n)$ and $V_{k}(n)$ can be expressed, in an additive way, by the corresponding expectations $m_{i} \ell_{i} / 2$, and variances $m_{i} \ell_{i}\left(\ell_{i}+2\right) / 12$, respectively.

In the univariate case we have: $E_{1}(n)=m \ell / 2$ and $V_{1}(n)=m \ell(\ell+2) / 12$

We conclude this section by observing the recurrence properties of eq. (12). Indeed $I(\bar{m}, n, \bar{\ell})$ can be recasted as, 


$$
\begin{aligned}
& I(\bar{m}, n, \bar{\ell})=\sum_{n_{k}=0}^{n} I\left(\bar{m}_{1}, n-n_{k}, \bar{\ell}_{1}\right) \cdot I\left(m_{k}, n_{k}, \ell_{k}\right), \bar{m}_{1} \\
& =\left(m_{1}, \ldots, m_{k-1}\right), \quad \bar{\ell}_{1}=\left(\ell_{1}, \ldots, \ell_{k-1}\right)
\end{aligned}
$$

For example, in the bivariate case, we have

$$
I_{2}\left(m_{1}, m_{2}, n, \ell_{1}, \ell_{2}\right)=\sum_{n_{2}=0}^{n} I\left(m_{1}, n-n_{2}, \ell_{1}\right) I\left(m_{2}, n_{2}, \ell_{2}\right)
$$

Note that calculation of eq. (12), which may need excessive computations, may be shortened by recalling that $I\left(m_{i}, n_{i}, \ell_{i}\right)$ vanishes if $n_{i}>m_{i} \ell_{i}$.

\section{GRAPHIC DISPLAYS}

In this work all graphic displays are presented as $Y$ (vertical axis) vs. $X$ (horizontal axis).

The following graphic output shows that the first gate distribution has a Gaussian fit in a wide range, including small values of $m$ and $\ell$.

Fig. (1) shows a plot as dots of $g_{n}(10, n, 3)$ and Fig. (2) of $g_{n}(4, n, 2)$. The solid line is a Gaussian fit using $E(n)$ and $V(n)$ as per eqs.(18) and (24) for $k=1$. Despite the fact that, unlike the Gaussian fit, $g_{n}(m, n, \ell)$ is defined in the range $0 \leq n \leq m \ell$, the fit is strong, even in values of $m$ and $\ell$ as small as 4 and 2, respectively. As expected the fit improves as $m$ and $\ell$ increase.

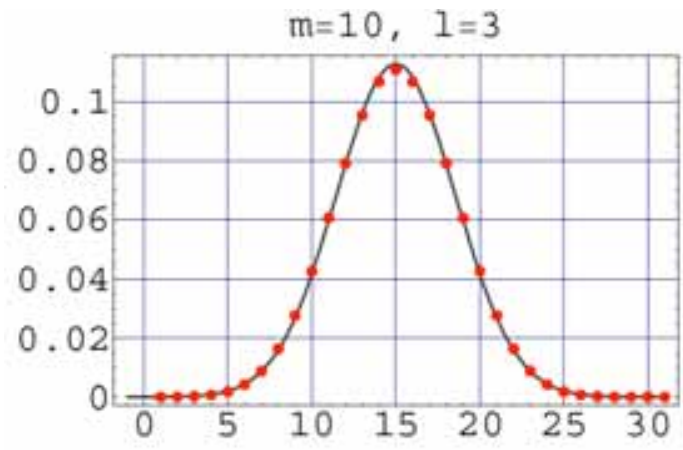

Fig. (1). A plot of the first gate distribution $g n(10 ; n ; 3)$ vs. $n$.

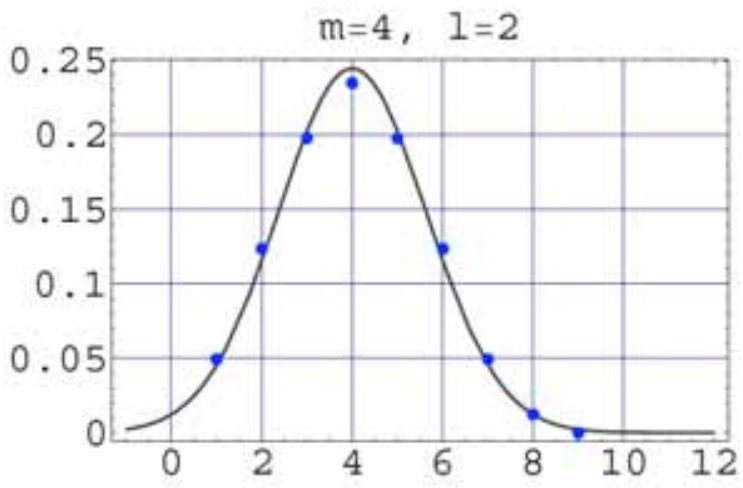

Fig. (2). A plot of the first gate distribution $g_{\mathrm{n}}(4 ; n ; 2)$ vs. $n$.
Fig. (3) shows, from left to right, dotted plots of $g_{n}\left(m_{1}, n_{1}, \ell_{1}\right), g_{n}\left(m_{2}, n-n_{1}, \ell_{2}\right)$, and $g_{n}\left(m_{1}, m_{2}, n, \ell_{1}, \ell_{2}\right)$ obtained as convolution of the first two: $m_{1}=10, \ell_{1}=3, m_{2}=20, \ell_{2}=4$.

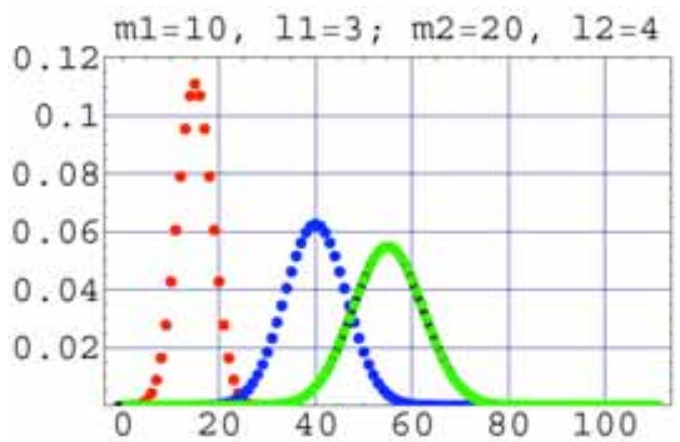

Fig. (3). A plot (from left to right) of two first gate distributions $g_{\mathrm{n}}(10 ; n ; 3)$ and $g_{\mathrm{n}}(20 ; n ; 4)$, and their convolution $g_{\mathrm{n}}(10 ; 20 ; n ; 3$; 4) vs. $n$.

A Gaussian fit (solid line) is shown for $g_{n}\left(m_{1}, m_{2}, n, \ell_{1}, \ell_{2}\right)$. Clearly the symmetry property, e.g. around $\frac{1}{2}\left(m, \ell_{1}+m_{2}, \ell_{2}\right)$, persists also in the bivariate first gate distribution, and the Gaussian fit is good. Fig. (4) shows a plot of $G_{\ell}(m, n, \ell)$ as a function of $\ell, n=350, m=2,5,7$, $10,14,25$ from right to left ( $m=2$, right most, straight line). The greater $m$ is the steeper is the slope of $G_{\ell}(m, n, \ell)$, which possesses a unique self shifting property of the range wherein it is defined. This range is $\frac{n}{m}-1 \leq \ell \leq n$, so that at $\ell=(n-m) / m, G_{\ell}(m, n, \ell)=0$ and at $\ell=n$, it is 1 .

Fig. (5) shows a plot of $g_{\ell}(m, n, \ell)$ derived from Fig. (4). Here $m$ increases from left to right, $m=2,5,7,10,14,25$ and $n=350$.

The second gate distribution is skewed to the right. The unique self shifting property is clearly displayed. Increase of $m$ shifts the left hand boundary of the distribution further to the left, and decreases its variance, and skewness coefficient. The maxima of the plots traces an increasing function of $m$.

Fig. (5) can be used to determine the effect of a unit change in $\ell$ on the capacity of the gate array at fixed number of gates $m$ and passing objects $n$. If $\ell=\frac{n}{m}$ or $\ell=n$, then a unit change in $\ell$ is the smallest, and may not justify the effort or cost. However, if $\ell$ is at the maximum of a given curve in Fig. (5), then a unit change in it is expected to produce a maximal effect in changing the number of ways the $n$ objects can pass the $m$ gates.

Consider the case where $\ell=2 \frac{n}{m}$ so that $g_{n}$ is at its peak. This gives $\ell=350,140,100,70,50,28$ for the curves in Fig. (5), from right to left, respectively. In the rightmost 


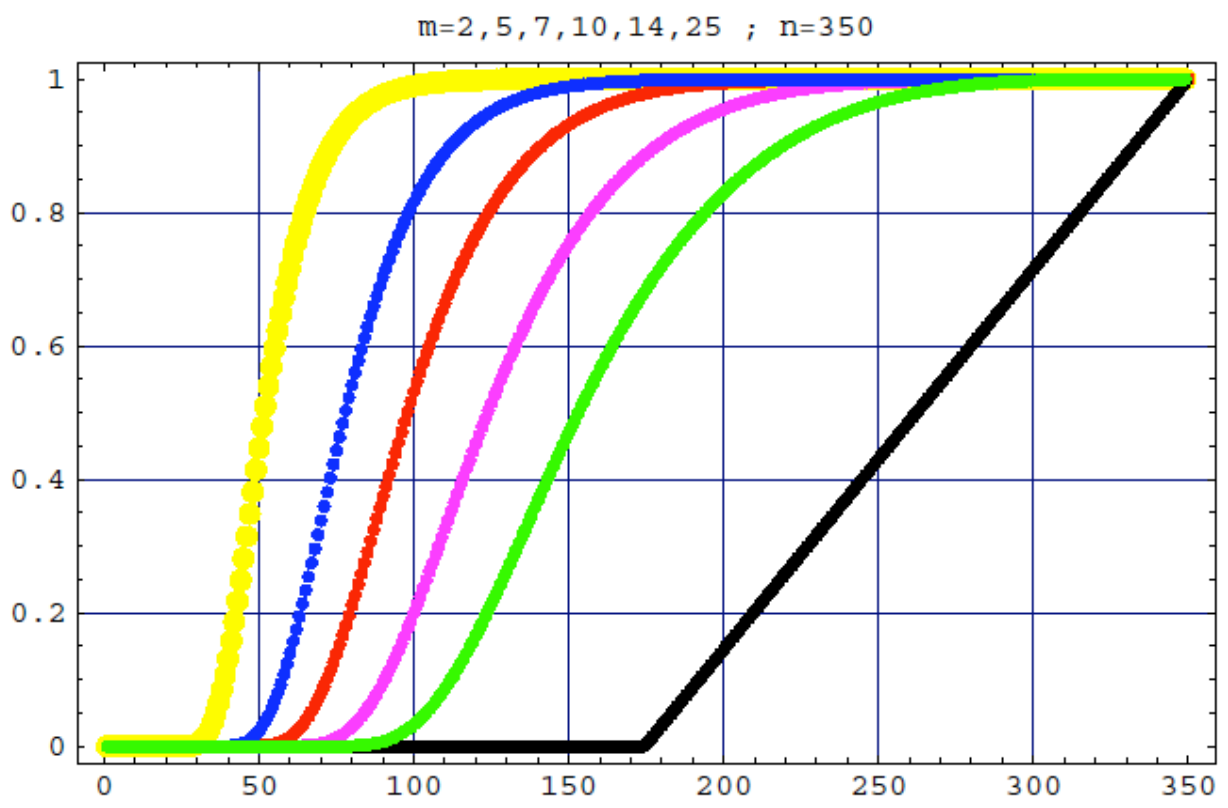

Fig. (4). A plot of $G_{l}(m ; n ; l)$ as a function of $l ; n=350, m=2 ; 5 ; 7 ; 10 ; 14 ; 25$. In the plot, $m$ increases from right to left.

horizontal line, $\ell=350$ is at the end of the range, where $g_{\ell}=0.05$. As the line is horizontal, all values of $\ell$ give the same results for the effect of its unit change. $\ell=140$ is close to the maximum in the second, $m=5$, distribution. As $\ell$ decreases, it shifts further to the left of the maximum. At $\ell=$ 50 ( $m=14$ curve), the maximum is at 70 , whereas at $\ell=28$ ( $m=25$ curve), the maximum is at 45 and the effect of a unit change in $\ell$ practically vanishes. Thus, the larger $m$ is, at fixed $n$, the smaller is the effect of a unit change in $\ell$ at the point where $n=\frac{1}{2} m \ell$. Here the effect of a unit change in $\ell$ intensifies as $\ell$ is increased, until the maximum in the distribution is reached. As $m$ increases, so does the value of the maximum in the corresponding distribution. Concurrently, the standard deviation decreases.
Plots of $G_{\ell}\left(m_{1}, m_{2}, n, \ell_{1}, \ell_{2}\right) v s . \ell_{1}$ and $\ell_{2}$ for $m_{1}=5, m_{2}$ $=10$, and for three different pairs of $m_{1}, m_{2}$ and $n=100$ are shown in Figs. (6), and (7), respectively. At high levels of $m_{1}=10, m_{2}=20$ and $n=100$, the top plot reaches rapidly an asymptotic range wherein it tends to unity. All three surfaces show that it is a monotonically increasing function of both $\ell_{1}$ and $\ell_{2}$. Smaller values of $m_{1}=5$ and $m_{2}=10$, intermediate (surface) and $m_{1}=2, m_{2}=5$ bottom surface, produce smaller slopes and hence, a larger variance of $g_{\ell}\left(m_{1}, m_{2}, n, \ell_{1}, \ell_{2}\right) w$. The self shifting property persists also here. Larger $m_{1}$ and $m_{2}$ shift the range boundary toward the origin.

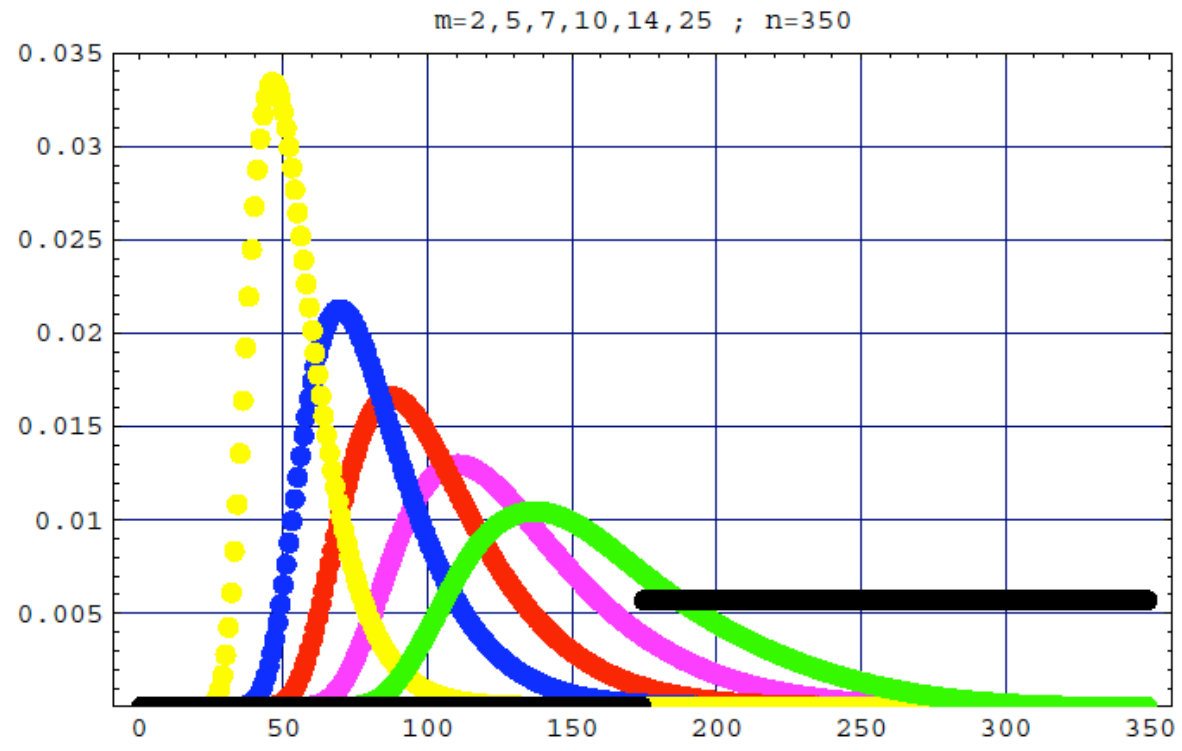

Fig. (5). A plot of $g_{l}(m ; n ; l)$ as a function of $l$, derived from Fig. (4). In the plot, $m$ increases from right to left. 


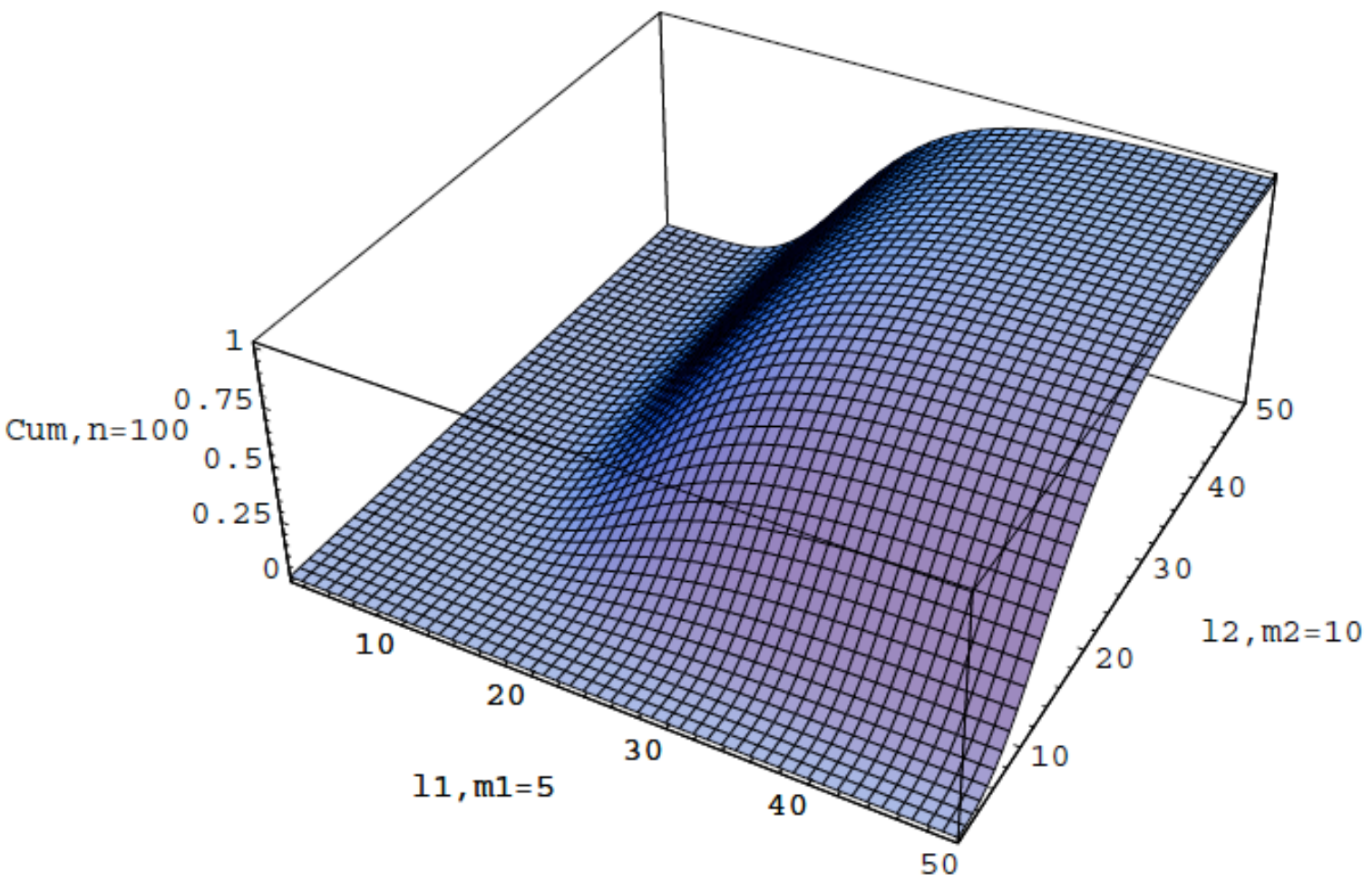

Fig. (6). A plot of $G_{1}\left(m_{1}, m_{2}, n, l_{1}, l_{2}\right) v s . l_{1}$ and $l_{2}$ for $n=100, m_{1}=5$ and $m_{2}=10$.

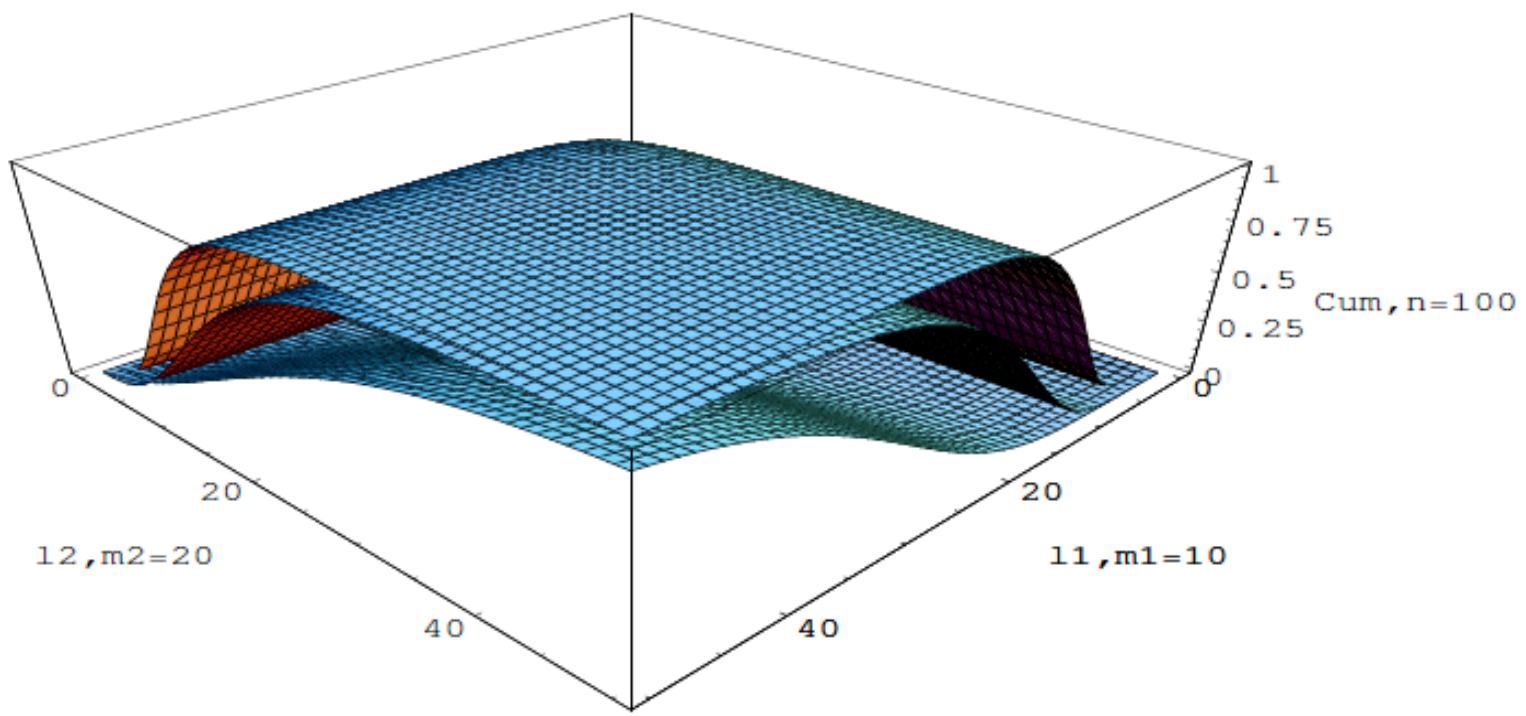

Fig. (7). A plot of $G_{1}\left(m_{1}, m_{2}, n, l_{1}, l_{2}\right) v s . l_{1}$ and $l_{2}$ at $n=100$ for three different pairs of $m_{1}, m_{2}$ : the upper surface $m_{1}=10, m_{2}=20$, the intermediate surface $m_{1}=5, m_{2}=10$, the lower surface $m_{1}=2, m_{2}=5$.

\section{APPENDIX}

Using the notation of Riordan [1], we define,

$$
E(t ; m, \ell)=\left(1+t+\ldots .+t^{\ell}\right)^{m}=\frac{\left(1-t^{\ell+1}\right)^{m}}{(1-t)^{m}}
$$

$$
\begin{aligned}
& (1-t)^{-m}=\sum_{u=0}^{\infty}\left(\begin{array}{c}
m+u-1 \\
u
\end{array}\right) t^{u},\left(1-t^{\ell+1}\right)^{m} \\
& =\sum_{k=0}^{m}(-1)^{k}\left(\begin{array}{c}
m \\
k
\end{array}\right) t^{k(\ell+1)}
\end{aligned}
$$

Expansion of the numerator and denominator gives,

Hence 
$E(t ; m, \ell)=\sum_{u=0}^{\infty} \sum_{k=0}^{m}(-1)^{k}\left(\begin{array}{c}m \\ k\end{array}\right)\left(\begin{array}{c}m+u-1 \\ u\end{array}\right) t^{u+k(\ell+1)}$

Denoting $u=n-k(\ell+1) \geq 0$, changes the $(k, u)$ variables to $(k, n)$, which are running in the range: $0 \leq k \leq\left\lfloor\frac{n}{\ell+1}\right\rfloor, 0 \leq n<\infty$. Thus,

$E(t ; m, \ell)=\sum_{n=0}^{\infty} I(m, n, \ell) t^{n}$

$I(m, n, \ell)=\sum_{k=0}^{\lfloor n /(\ell+1)\rfloor}(-1)^{k}\left(\begin{array}{l}m \\ k\end{array}\right)\left(\begin{array}{c}m+n-k(\ell+1)-1 \\ n-k(\ell+1)\end{array}\right)$.

Equations (A5) and (1) are identical except for difference in notation.

The number of ways that a queue of up to $n-\ell$ objects can be formed in at least one of the $\mathrm{m}$ gates is,

$$
\begin{aligned}
& Q(m, n, \ell)=\left(\begin{array}{c}
m+n-1 \\
m-1
\end{array}\right) I(m, n, \ell) \\
& =\sum_{k=1}^{n /(\ell+1)\rfloor}(-1)^{k-1}\left(\begin{array}{l}
m \\
k
\end{array}\right)\left(\begin{array}{c}
m+n-k(\ell+1)-1 \\
m-1
\end{array}\right)
\end{aligned}
$$

Represent the generating polynomial $E(t ; m, \ell)$ in $t$ as follows,
$E(t ; m, \ell)=\left(\sum_{k=0}^{\ell} t^{k}\right)^{m}=\sum_{n=0}^{m \ell} I(m, n, \ell) t^{n}$,

and recast the last as

$$
E(t ; m, \ell)=t^{m \ell} E\left(t^{-1} ; m, \ell\right)
$$

A straightforward calculation in (A8) gives,

$$
t^{m \ell} E\left(t^{-1} ; m, \ell\right)=\sum_{n=0}^{m \ell} I(m, n, \ell) t^{m \ell-n},
$$

and substituting $w=m \ell-n$, leads (after reversing the summation) to

$$
t^{m \ell} E\left(t^{-1} ; m, \ell\right)=\sum_{w=0}^{m \ell} I(m, m \ell-w, \ell) t^{w}
$$

By virtue of eqs. (A7) and (A9), we get

$$
I(m, n, \ell)=I(m, m \ell-n, \ell)
$$

\section{REFERENCES}

[1] Riordan J. An introduction to combinatorial analysis. Princeton, N.J.: Princeton University Press, 1980

[2] Brualdi RA. Introductory combinatorics. $2^{\text {nd }}$ ed. Englewood Cliffs, N.J.: Prentice-Hall, 1992.

[3] Papoulis A. Probability, random variables, and stochastic processes. $3^{\text {rd }}$ ed. New York: McGraw-Hill, 1991.

[4] Liu CL. Introduction to combinatorial mathematics. New York: McGraw-Hill, 1968. 